\title{
Chimera States: The Natural Link Between Coherence and Incoherence
}

\author{
Oleh E. Omel'chenko, ${ }^{1,2,4}$ Yuri L. Maistrenko, ${ }^{1,4}$ and Peter A. Tass ${ }^{1,3}$ \\ ${ }^{1}$ Institute of Neuroscience and Biophysics 3-Medicine, Research Center Jülich, 52425 Jülich, Germany \\ ${ }^{2}$ Department of Mathematics, Humboldt University of Berlin, 10099 Berlin, Germany \\ ${ }^{3}$ Department of Stereotaxic and Functional Neurosurgery, University of Cologne, 50924 Cologne, Germany \\ and Brain Imaging Center West, 52425 Jülich, Germany \\ ${ }^{4}$ Institute of Mathematics, National Academy of Sciences of Ukraine, 01601 Kyiv, Ukraine
}

(Received 8 November 2007; published 31 January 2008)

\begin{abstract}
Chimera states are remarkable spatiotemporal patterns in which coherence coexists with incoherence. As yet, chimera states have been considered as nongeneric, since they emerge only for particular initial conditions. In contrast, we show here that in a network of globally coupled oscillators delayed feedback stimulation with realistic (i.e., spatially decaying) stimulation profile generically induces chimera states. Intriguingly, a bifurcation analysis reveals that these chimera states are the natural link between the coherent and the incoherent states.
\end{abstract}

DOI: 10.1103/PhysRevLett.100.044105

PACS numbers: 05.45.Xt, 89.75.Kd

Synchronization processes are of great importance in various fields of physics, chemistry, biology, and medicine [1-4]. Numerous studies have been devoted to the transition from incoherent to synchronization regimes $[1,2,5]$. In recent years in a series of papers a strange new mode of organization in arrays of identical limit-cycle oscillators has been reported [6-9]. This peculiar mode, in which coherence and incoherence coexist at the same time in a system of oscillators, was first noticed by Kuramoto and colleagues [6-8] in their simulations of the complex Ginzburg-Landau equation with nonlocal coupling, and was later called a chimera state by Abrams and Strogatz [9]. The emergence of the chimera state was ascribed by all authors to the impact of nonlocal coupling and was, thus, considered to depend on intrinsic parameters of the system. Moreover, all known chimera states coexist with a linearly stable coherent state, and therefore one requires a very specific initial condition to approach them. The chimera states revealed so far are nongeneric in the sense that they occur only for particularly prepared initial conditions.

In this Letter we describe how chimera states emerge due to delayed feedback, which is a major approach to controlling or manipulating synchronization processes in ensembles of oscillators [10-15]. We show that chimera states generically emerge already in a rather simple network of globally coupled oscillators, provided the latter is subject to spatially modulated delayed feedback. Spatial modulation here means that the strength of the delayed feedback is maximal at the site of injection and decreases with increasing distance from the injection site. This feature is typical for spatially extended systems under the influence of nonhomogeneous, local control forces [16]. Remarkably, in our model the emergence of a chimera state depends mainly on the amplification and the delay in the feedback loop, and these two parameters are well adjustable in real experimental setups. Moreover, there is a sufficiently broad parameter range where the chimera states do not coexist with any stable synchronized state.
In that region the chimera state emerges out of the coherent initial condition, which is both the natural state of our model system and a typical initial state in numerous control studies [10-12,14,15].

We start with an ensemble of identical, densely and uniformly distributed Landau-Stuart oscillators, representing a normal form of a supercritical Andronov-Hopf bifurcation. The oscillators are globally coupled and exposed to a delayed feedback stimulation according to

$$
\frac{\partial W}{\partial t}=\left(1+i \omega-|W|^{2}\right) W+C[\bar{W}(t)-W]+S(x, t) .
$$

Here $W(x, t)$ stands for a complex amplitude of the oscillator at position $x$ at time $t$. Our approach is valid for any space dimension and any measurable space domain, but for the sake of simplicity below we assume that $x$ is one dimensional and runs from -1 to 1 . Positive parameters $\omega$ and $C$ denote the natural frequency of the oscillators and the global coupling strength, respectively, $\bar{W}(t)=\frac{1}{2} \times$ $\int_{-1}^{1} W(x, t) d x$ is the ensemble's mean field. We assume in Eq. (1) that the mean field is delivered with the delay $\tau$ and the spatial profile $\rho(x)$ to each oscillator according to $S(x, t)=K \rho(x) \bar{W}(t-\tau)$, where the constant $K$ is the strength of the delayed feedback. The stimulation profile $\rho(x)$ is a non-negative even function, strictly decreasing on $[0,1]$ and obeying the normalization condition $\frac{1}{2} \times$ $\int_{-1}^{1} \rho(x) d x=1$.

For weak coupling and control, the oscillators' amplitudes can be eliminated $(|W(x, t)| \rightarrow 1)$ and the complex Eq. (1) is reduced $[1,17]$ to the scalar equation of the phase dynamics

$$
\begin{aligned}
\frac{\partial \psi}{\partial t}= & \omega-\frac{C}{2} \int_{-1}^{1} \sin (\psi(x, t)-\psi(\xi, t)) d \xi \\
& -\frac{K}{2} \int_{-1}^{1} \rho(x) \sin (\psi(x, t)-\psi(\xi, t-\tau)) d \xi,
\end{aligned}
$$


where $\psi(x, t)$ is the phase of the oscillator at position $x$ at time $t$. To assess the impact of the particular shape of the stimulation profile, we use in Eq. (2) the exponential stimulation profile

$$
\rho(x)=a e^{-a|x|} /\left(1-e^{-a}\right) \quad \text { with } \quad a>0 .
$$

The discrete analog of Eq. (2) with spatially uniform stimulation profile $[\rho(x) \equiv 1]$ has been studied in detail with zero coupling $(C=0)$ [10] and with nonzero coupling $(C>0)$ [11]. In both cases only completely coherent or completely incoherent stable solutions were observed. Remarkably, the stability analysis of the incoherent solution developed in $[10,11]$ is also applicable to Eq. (2) with spatially nonuniform stimulation profile. Moreover, due to the normalization condition $\frac{1}{2} \int_{-1}^{1} \rho(x) d x=1$, we obtain the same analytical expressions for the boundaries of the stability region. Some results concerning the existence and stability of only the coherent solutions of Eq. (2) with nonconstant stimulation profile were presented in $[14,15]$. Numerical simulations of Eq. (2) with parameters chosen such that neither a stable coherent nor a stable incoherent solution exist yield chimera states (Fig. 1). In the chimera states the ensemble of identical oscillators is split into two domains: In the domain close to the apex of the stimulation profile the oscillators are coherent and phase locked. In contrast, in the other domain the oscillators are incoherent and desynchronized. It occupies the rest of the interval $[-1,1]$, where the stimulation impact falls below a certain threshold. Plotting the effective frequencies of the oscillators, $\omega_{\text {eff }}(x)=\lim _{t \rightarrow \infty} \frac{1}{t} \int_{0}^{t} \dot{\psi}(x, s) d s$, we observe that in the coherent domain they are identical, while in the inco-
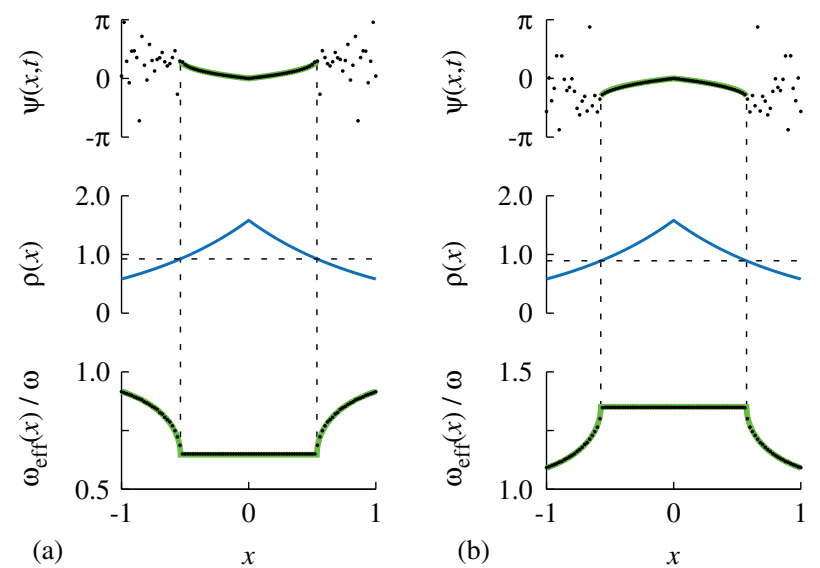

FIG. 1 (color). Chimera states induced by the delayed feedback with the exponential stimulation profile (3). Parameters: $a=1.0, \quad C=0.1 \pi, K=\pi, \omega=2 \pi$, and $\tau=0.3$ (a) or $\tau=0.6$ (b). Equation (2) was approximated with an array of 101 oscillators uniformly distributed over the interval $[-1,1]$ and was integrated using a Runge-Kutta method starting from the coherent initial condition. Top: Snapshot of the oscillators' phases (black dots). Middle: Stimulation profile. Bottom: Effective frequencies of the oscillators (black dots). Green thick lines in the top and bottom graphs represent the theoretical predictions obtained from Eqs. (6)-(8). herent domain they are nonidentical but lie on a welldefined continuous curve. We distinguish two types of chimeras depending on whether the effective frequencies of the incoherent oscillators are greater [Fig. 1(a)] or smaller [Fig. 1(b)] than the frequencies of the coherent ones, denoted as chimera I and chimera II, respectively.

To explain these numerical results we utilize an approach similar to that proposed by Kuramoto and Battogtokh [6]. For this we introduce the relative phase $\theta$ by $\theta=\psi-\Omega t$, where $\Omega$ denotes the angular frequency of an appropriate rotating frame in which the dynamics gets simplified. Accordingly, $\Omega$ is the effective frequency of the oscillators in the coherent domain. We define a complex order parameter with modulus $R$ and phase $\Theta$

$$
R(t) e^{i \Theta(t)}=\frac{1}{2} \int_{-1}^{1} e^{i \theta(x, t)} d x
$$

where $0 \leq R(t) \leq 1$ holds for all times $t$, and $R=1$ or $=0$ corresponds to perfect in phase synchronization or absence of in phase synchronization. Then Eq. (2) reads

$$
\frac{\partial \theta}{\partial t}=\omega-\Omega-C R \sin \theta(x, t)-K R \rho(x) \sin (\theta(x, t)+\Omega \tau) .
$$

Following Kuramoto and Battogtokh [6], we study the existence of statistically stationary solutions of Eq. (5) for which the long-term averaged values of $R$ and $\Theta$ are time independent. In contrast to their method for nonlocally coupled oscillators, we assume here that the averaged values of $R$ and $\Theta$ are also space independent, i.e., constants. Given $R$ and $\Omega$, one can then easily identify coherent and incoherent domains with the function

$$
g(x, R, \Omega)=\frac{\omega-\Omega}{R \sqrt{[C+K \rho(x) \cos (\Omega \tau)]^{2}+[K \rho(x) \sin (\Omega \tau)]^{2}}} .
$$

If $|g(x, R, \Omega)| \leq 1$, then Eq. (5) has a stable fixed point

$$
\theta(x, R, \Omega)=-\alpha(x, R, \Omega)+\sin ^{-1} g(x, R, \Omega),
$$

where $\alpha(x, R, \Omega)$ is uniquely defined by the relation

$$
e^{i \alpha}=\frac{C+K \rho(x) \cos (\Omega \tau)+i K \rho(x) \sin (\Omega \tau)}{\sqrt{[C+K \rho(x) \cos (\Omega \tau)]^{2}+[K \rho(x) \sin (\Omega \tau)]^{2}}} .
$$

This fixed point stands for the time-independent asymptotic phase deviation of a synchronized oscillator with respect to the macroscopic phase $\Theta$. In contrast, oscillators for which $|g(x, R, \Omega)|>1$ do not reach a stationary deviation from the macroscopic phase and, therefore, do not synchronize. Replacing now in definition (4) the factor $\exp (i \theta(x, t))$ by its statistical average, and calculating the latter with the help of expression (6) on the coherent interval and with the help of an invariant measure obtained from Eq. (5) on the incoherent interval (see details in [6]), we derive a self-consistency equation 


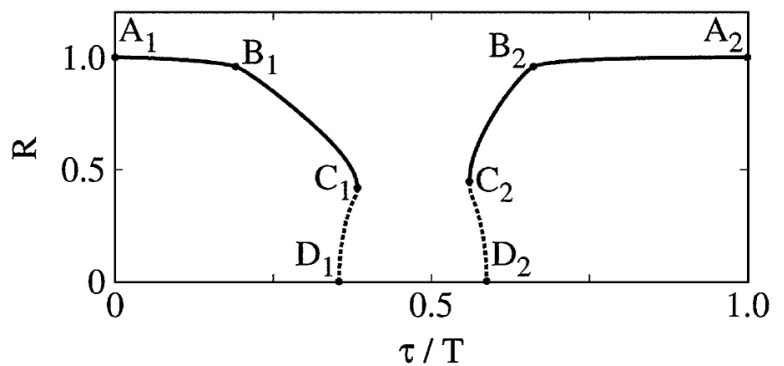

FIG. 2. Order parameter $R$ from Eq. (7). Parameters: $C / \omega=$ $0.05, K / \omega=0.5$, and exponential stimulation profile (3) with $a=1.0$. Solid lines represent stable branches of the solution: phase-locked state $\left(\operatorname{arcs} A_{1} B_{1}\right.$ and $\left.A_{2} B_{2}\right)$, chimera I $\left(\operatorname{arc} B_{1} C_{1}\right)$ and chimera II $\left(\operatorname{arc} B_{2} C_{2}\right)$. Dashed lines $C_{1} D_{1}$ and $C_{2} D_{2}$ stand for unstable branches. The completely incoherent state is stable for delays taken from the interval $D_{1} D_{2}$.

$$
\begin{aligned}
& \frac{1}{2} \int_{-1}^{1} e^{-i \alpha(x, R, \Omega)} H(g(x, R, \Omega)) d x=R, \\
& H(g)= \begin{cases}\sqrt{1-g^{2}}+i g & |g| \leq 1 \\
i\left\{g-\operatorname{sgn}(g) \sqrt{g^{2}-1}\right\} & |g|>1 .\end{cases}
\end{aligned}
$$

In fact, Eq. (7) is a system of two real equations for the two unknowns $R$ and $\Omega$. This is significantly simpler than solving a functional self-consistency equation for chimera states in a system of nonlocally coupled oscillators [6]. For Eq. (7) the position of the critical points separating the coherent and the incoherent domains is given by $|g(x, R, \Omega)|=1$. In the coherent domain the effective frequencies of the oscillators equal $\Omega$, while in the incoherent domain they obey

$$
\omega_{\mathrm{eff}}(x)=\Omega+(\omega-\Omega) \sqrt{1-g^{-2}(x, R, \Omega)} .
$$

The theoretical curves obtained from Eqs. (6)-(8) fit perfectly to the numerical simulations of Eq. (2) (Fig. 1). This shows that Eq. (7) is an adequate model capturing the chimera states. Moreover, if $|g(x, R, \Omega)| \leq 1$ holds for all $x \in[-1,1]$, then Eq. (7) coincides with system (19) from [15], which was shown to yield phase-locked solutions of Eq. (2). Consequently, in a certain sense the chimera states are linked to the coherent, phase-locked, solutions of Eq. (2). This motivates us to solve Eq. (7) numerically, to reveal solutions which constitute a continuous extension of the completely coherent state $(R=1.0, \Omega=\omega)$. The latter obviously satisfies Eq. (7) for $\tau=k T, k=$ $0, \pm 1, \pm 2, \ldots$, where $T=2 \pi / \omega$ is the natural period of the oscillation [18]. The phase-locked and chimera states obtained from Eq. (7) were used as initial conditions in an extensive series of numerical simulations of a noisy discrete approximation of Eq. (2) (with additive Gaussian white noise). These simulations were used to verify the results from Eq. (7) and to check their stability.

A typical solution of Eq. (7) is shown in Fig. 2. The starting point $A_{1}$ is the completely coherent state. With increasing $\tau / T$ this state, first, survives as a stable phaselocked solution with modified effective frequency and deformed phase pattern for delays up to a certain critical value (see [15]). Point $B_{1}$ indicates the boundary between the phase-locked solution and the chimera state. For larger delays one observes a series of stable chimera states along arc $B_{1} C_{1}$ (the curve from $B_{1}$ to $C_{1}$ ). We characterize their "chimerness" using the synchronization parameter defined as the relative measure of the coherent domain. It varies between 0 and 1 , and equals 1 for phase-locked solutions only. Along arc $B_{1} C_{1}$ the synchronization parameter decreases monotonically with increasing delay, which corresponds to a progressive "evaporation" of the oscillators from the phase-locked to the drifting state. At point $C_{1}$,


FIG. 3 (color). (a),(b) Domains in the control parameters plane with one (yellow) or two (orange) stable chimera states. The incoherent state is stable inside the regions marked by blue lines. At least one stable coherent solution exists in the region outside of the green lines (see enlargement in Fig. 4). Parameters: exponential stimulation profile with $a=$ 1.0 and $C / \omega=0.05$ (a) or $C=0$ (b). Synchronization parameter of the chimera I (c) and chimera II (d) belonging to (a). 


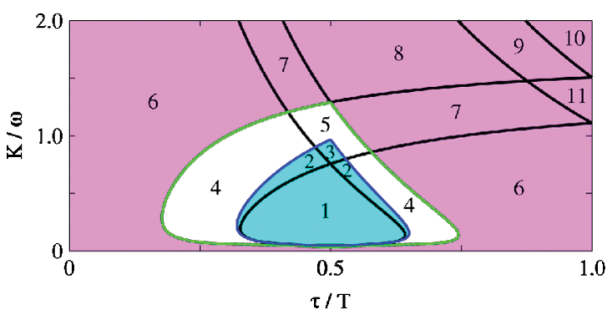

FIG. 4 (color). The enlarged left third of Fig. 3(a) shows the linkage between incoherent states (blue) and coherent states (violet). The incoherent state is stable in the blue region. At least one stable coherent solution exists in the violet region. There is a small white gap between the blue and violet region even at the bottom at $\tau / T \approx 0.5$ : Incoherent and coherent states are not directly transformed into one another. Numbers in the plot indicate different types of stable solutions: incoherence (1), incoherence and chimera (2), incoherence and two chimeras (3), chimera (4), two chimeras (5), coherent solution (6), coherent solution and chimera (7), two coherent solutions (8), two coherent solutions and chimera (9), three coherent solutions (10), two coherent solutions and two chimeras (11).

where the synchronization parameter reaches a certain threshold, a saddle-node bifurcation occurs: the stable chimera collides with an unstable solution, which can be traced towards a subcritical bifurcation point $D_{1}$, at which the completely incoherent state $(R=0)$ changes its stability. Between $D_{1}$ and $D_{2}$ the incoherent state is stable. An analogous scenario is observed along the curve $A_{2} B_{2} C_{2} D_{2}$. The only difference is that the $\operatorname{arcs} B_{1} C_{1}$ and $B_{2} C_{2}$ correspond to chimera I and chimera II, respectively.

Taking into account Fig. 2, with the same analyticnumerical technique as described above, we revealed the main features of the complete solution of Eq. (7) [Figs. 3(a), 3(c), and 3(d)]. Note the pronounced multistability, where different values of the control parameters $\tau / T$ and $K / \omega$ may be connected with different combinations of coexisting stable solutions (Fig. 4). For control parameters taken from the white region in Fig. 4 the chimera state is a unique stable solution, which evolves starting from the coherent initial condition, i.e., from the natural state of the unperturbed globally coupled system.

The results presented above are robust with respect to variations of the coupling strength and the shape of the stimulation profile. This can be seen, e.g., by comparing Fig. 3(a) with the analogous Fig. 3(b) for vanishing coupling $(C=0)$ or with the corresponding results for the linear stimulation profile (data not shown).

In summary, in a network of globally coupled oscillators chimera states can robustly be induced by delayed feedback stimulation with a variety of exponentially or linearly decaying stimulation profiles, provided the key stimulation parameters, the delay and strength, are tuned appropriately. Thus, already in a rather simple model with a realistic stimulation setup, chimera states are not just curious nongeneric solutions, but the natural link between coherent and incoherent states. Accordingly, chimera states may be relevant in other physical and biological systems too [19].

This study was supported by the Alexander von Humboldt Foundation (O. O.) and the EU Network of Excellence in Biosimulation LSHB-CT-20004-005137).

[1] Y. Kuramoto, Chemical Oscillations, Waves, and Turbulence (Springer, Berlin, 1984).

[2] S. H. Strogatz, Physica (Amsterdam) 143D, 1 (2000); A. Winfree, The Geometry of Biological Time (SpringerVerlag, Berlin, 2001); A. Pikovsky et al., Synchronization, A Universal Concept in Nonlinear Sciences (Cambridge University Press, Cambridge, 2001); E. Mosekilde et al., Chaotic Synchronization: Application to Living Systems (World Scientific, Singapore, 2002); P. A. Tass, Phase Resetting in Medicine and Biology (Springer-Verlag, Berlin, 1999).

[3] S. Boccaletti et al., Phys. Rep. 366, 1 (2002).

[4] G. Kozyreff et al., Phys. Rev. Lett. 85, 3809 (2000).

[5] P. Ashwin et al., Nonlinearity 9, 703 (1996); V. Belykh et al., Phys. Rev. E 62, 6332 (2000); W. Wang et al., Chaos 10, 248 (2000); Y. Maistrenko et al., Phys. Rev. Lett. 93, 084102 (2004); D. Pazo, Phys. Rev. E 72, 046211 (2005).

[6] Y. Kuramoto and D. Battogtokh, Nonlinear Phenom. Complex Syst. 5, 380 (2002).

[7] Y. Kuramoto, in Nonlinear Dynamics and Chaos: Where Do We Go from Here?, edited by S. J. Hogan et al. (Institute of Physics, Bristol, England, 2003), p. 209.

[8] S. I. Shima and Y. Kuramoto, Phys. Rev. E 69, 036213 (2004).

[9] D. M. Abrams and S. H. Strogatz, Phys. Rev. Lett. 93, 174102 (2004); D. M. Abrams and S. H. Strogatz, Int. J. Bifurcation Chaos Appl. Sci. Eng. 16, 21 (2006).

[10] M. K. S. Yeung and S. H. Strogatz, Phys. Rev. Lett. 82, 648 (1999).

[11] M. G. Rosenblum and A. S. Pikovsky, Phys. Rev. Lett. 92, 114102 (2004).

[12] O. Popovych et al., Phys. Rev. Lett. 94, 164102 (2005).

[13] N. Janson et al., Phys. Rev. Lett. 93, 010601 (2004); B. Hauschildt et al., Phys. Rev. E 74, 051906 (2006); A. Balanov et al., Phys. Rev. E 74, 016214 (2006).

[14] C. Hauptmann et al., Phys. Rev. E 76, 066209 (2007).

[15] O. Omel'chenko et al., Physica D (Amsterdam) doi:10.1016/j.physd.2007.09.019 (2008).

[16] N. Yousif and X. Liu, Expert Rev. Med. Dev. 4, 623 (2007).

[17] P. A. Tass and H. Haken, Z. Phys. B 100, 303 (1996).

[18] If $\Omega=\omega$ and $\omega \tau=2 \pi k, k \in \mathbb{Z}$, we obtain $g=\sin \alpha=$ $0, \cos \alpha=1$, and Eq. (7) yields $R=1$.

[19] A fundamental problem in neuroscience is feature binding [20]: the integration of information processed in different parts of the brain. Apart from the favored overall synchronization [20], chimeras, i.e., anatomically distributed welltuned mixtures of coherency and incoherency, might also be candidate mechanisms for binding.

[20] W. Singer and C. M. Gray, Annu. Rev. Neurosci. 18, 555 (1995). 